\title{
Study on energy transmission through metal wall based on piezoelectric material
}

\author{
Yun-zhe Sun ${ }^{1}$, Ding-xin Yang ${ }^{1}$, Zheng $\mathrm{Hu}^{1}$, Hai-feng $\mathrm{Hu}^{1}$ and Bao-jian $\mathrm{Hou}^{1}$ \\ ${ }^{1}$ Laboratory of science and technology on integrated logistics support, National University of Defense Technology, Hunan Changsha, \\ China
}

\begin{abstract}
The efficiency of electric energy transmission through metal wall by ultrasonic wave mainly depends on the relevant parameter of the sandwich structure system, which is consist of a transit piezoelectric transducer, a receive piezoelectric transducer and a metal wall. This paper discussed the characteristics measurement of piezoelectric energy transmission channel through metal wall based on a kind of piezoelectric transducer. First, the intrinsic impedance characteristic of piezoelectric transducer is measured using a high precision impedance analyzer. Then ultrasonic energy transmission channel through a metal wall testing system is analyzed. The voltage ratio under different frequencies of the channel is measured and the maximum energy transmission frequency is obtained. Mason model is verified and compared with the experimental results, which provides a beneficial reference for further researches on ultrasonic through metal wall energy transmission.
\end{abstract}

\section{Introduction}

Many sensing applications are used within metallic vessels to monitor extreme environmental conditions. With the disadvantages of feed-through wires, it proposed a great challenge to the energy supply system and the technology of transmitting energy through metal wall has become a hotspot. Under the influence of the Faraday shielding [1], traditional wireless electromagnetic energy could not transmit through metal barriers with no decay [2]. An alternative approach to transmit electric energy is ultrasonic which has perfect performance when through solid wall even it's a metal wall [3]. Because of its perfect performance in transmission capacity and power transmission efficiency, ultrasonic energy soon got the attention of the public [4]. Using signal generator can generate electric signal, which can be converted into ultrasonic vibrations by the transducer because of the inverse piezoelectric effect and then the ultrasonic waves penetrate the solid metal wall [5]. Simultaneously, the acoustic mechanical vibration energy of the metal wall can be received and converted into electric energy by the piezoelectric transducers [6] because of the piezoelectric effect, which provides a means for wireless energy transformation.

In order to efficiently transfer energy through a metal wall, it is important to modeling the acoustic-electric energy transmission channel. Numerous equivalent circuit theories have been developed. Most of the models so far described in the literature [7-9] have been used for only the simulation of the piezoelectric transducer. First, the channel of energy transmission of the theoretical analysis is introduced. Then the experimental of characteristics of through metal wall based on ultrasonic energy transmission channel are test. The experimental results and simulation results are discussed in last.

\section{Piezoelectric Transducer Impedance and Acoustic-Electric Energy Transmis sion Channels}

Piezoelectric ceramic can be made into piezoelectric devices for various purposes because of its piezoelectric effect. Using acoustic waves to transfer piezoelectric energy by transmit and receive piezoelectric transducer with the structure by coaxially aligning a pair of piezoelectric transducers on opposite sides of a metal barrier, just like a 'Sandwich', the structure described as shown in Fig. 1.Continuous-wave excite the outside transducer to create an acoustic beam which is captured by the inside transducer, forming an acoustic-electric link for power harvesting circuitry. 


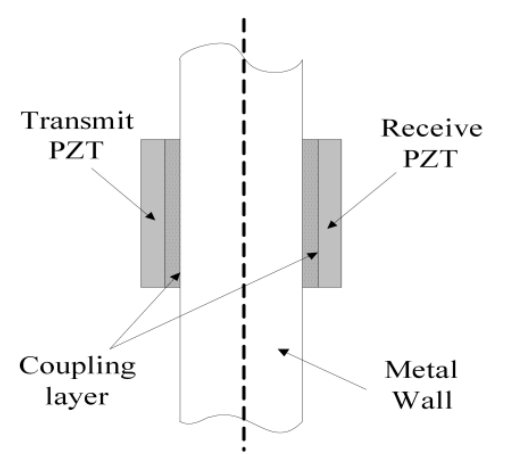

Figure 1. Structure of wireless energy transfer through metal wall

In the work of $\mathrm{Hu}[10]$ the piezoelectric and coupling layer was solved using the wave equation and linear equations of piezoelectricity. In order to eliminate the elements circuit between the top transformer and the node of the acoustic transmission and the first lumped equivalent circuit was proposed by Mason. The modeling of other theories based on equivalent circuits develop by Sherri [11] that can easily be simulated when connect to other circuits or networks. This modeling analysis conveniently simulates the dynamic behavior of the mechanical vibration and electricity conversion systems. The schematic of acoustic and electric equivalent circuit of Mason model is shown in Fig. 2.

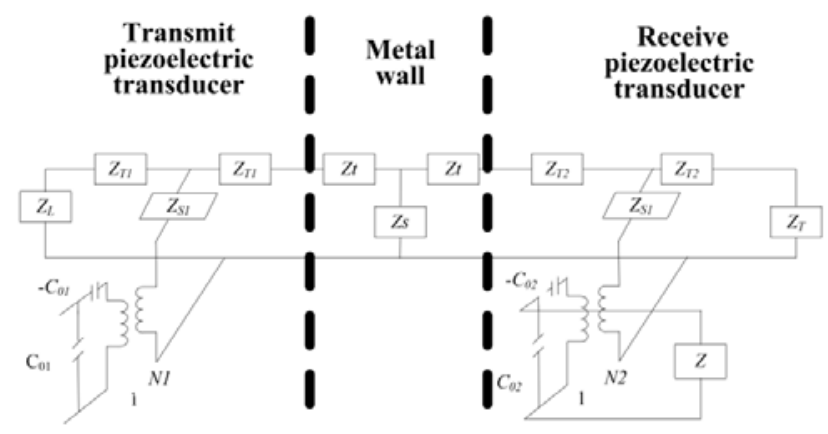

Figure 2. the schematic of acoustic and electric equivalent circuit of Mason model

The piezoelectric material parameters of modeling required are clamped complex permittivity $\varepsilon 33 \mathrm{~S}$, complex electromechanical coupling kt, and open circuit complex elastic stiffness c33D. Besides $\rho=$ density, $t=$ thickness, $\mathrm{A}=$ area.

$$
\begin{aligned}
& k_{t}^{2}=e_{33}^{2} / c_{33}^{D} \varepsilon_{33}^{s}=h_{33}^{2} \varepsilon_{33}^{s} / c_{33}^{D} \\
& h_{33}=k_{t} \sqrt{c_{33}^{D} / \varepsilon_{33}^{s}}
\end{aligned}
$$

Mason Model of transmit piezoelectric transducer

$$
\begin{gathered}
C_{01}=\frac{\varepsilon_{33_{1}}^{s} A_{1}}{t_{1}} \\
N_{1}=C_{01} h_{33_{1}}
\end{gathered}
$$

$$
\begin{aligned}
& Z_{01}=\rho_{1} A_{1} V_{1}^{D}=A_{1} \sqrt{\rho_{1} \varepsilon_{33_{1}}^{S}} \\
& \Gamma_{1}=\frac{\omega}{V_{1}^{D}}=\omega \sqrt{\frac{\rho_{1}}{c_{33_{1}}^{D}}} \\
& Z_{T 1}=i Z_{01} \tan \left(\Gamma_{1} t_{1} / 2\right) \\
& Z_{S 1}=-i Z_{01} \csc \left(\Gamma_{1} t_{1}\right)
\end{aligned}
$$

The configuration shown as Fig. 2 can also apply to voltage transformers and the model proceeds as with any network solution. In this approach the voltage on mechanical side of a transformer corresponds with the velocity of the surface. Voltage is multiplied by a factor of $\mathrm{N}$ when in the process of conversion of electrical energy to mechanical energy. Accordingly, Reverse process have to be divided by a ratio N. Starting from the model of equivalent circuit loads to the left, the impedance is as the following

$$
\begin{aligned}
& Z x 1=Z \frac{Z 02}{Z+Z 02} \\
& Z \times 2=Z_{x} 1-Z 02 \\
& Z_{X} 3=N_{2}^{2} Z_{x} 2 \\
& Z_{X} 4=Z_{s} 2+Z_{x} 3 \\
& Z_{X} 5=Z_{X} 4 \frac{(Z t 2+Z t R)}{\left(Z t 2+Z t R+Z_{X} 4\right)} \\
& Z X 6=Z_{X} 5+Z t 2+Z t w \\
& Z_{X} 7=Z_{X} 6 \frac{Z_{S W}}{Z_{X} 6+Z_{S W}} \\
& Z_{X} 8=Z_{X} 7+Z t_{W}+Z t 1 \\
& Z_{X} 9=Z_{x} 8 \frac{(Z t 1+Z t L)}{(Z t 8+Z t 1+Z t L)} \\
& Z_{X} 10=Z_{X} 9+Z_{S} 1 \\
& Z_{X} 11=\frac{Z_{X} 10}{N_{1}^{2}} \\
& Z_{X} 12=Z_{X} 11-Z 01
\end{aligned}
$$




$$
Z_{X} 13=Z_{X} 12 \frac{Z 01}{\left(Z_{X} 12+Z_{X} 01\right)}
$$

Where the Zx13 is electrical impedance of the transmit transducer. According to the above formulas a variety of equivalent impedance can be calculated. Note that is the impedance value is dynamic changes because the magnitude and the phase of sinusoidal input changes.

\section{Experiments and Simulation}

The system of ultrasonic energy transmission through metal wall is consists of a signal generator of TEKRONIX-AFG302, the channel of metal wall coupling with piezoelectric transducers and oscilloscope of TEKTRONIX-TDS2012B. The detail of the experimental process as following:

Running the software of Agilent Connection Expert which will auto search the net port connecting to the PC and indication it. Then, we refresh the tmtool toolbox software of MATLAB and click the connect button to get handler functions. This protocol realizes effective and reliable communication between debugger and monitor and simplifies their design. In this system, the signal can be controlled by programming on PC, which gives full play to the advantage of the computer, by this way by using MATLAB software to control signal generator to generate sweeping frequency excitations. Meanwhile, we get the graph of frequency response received by oscilloscope. Operation schematic diagram of experimental process is shown in Fig. 3 .

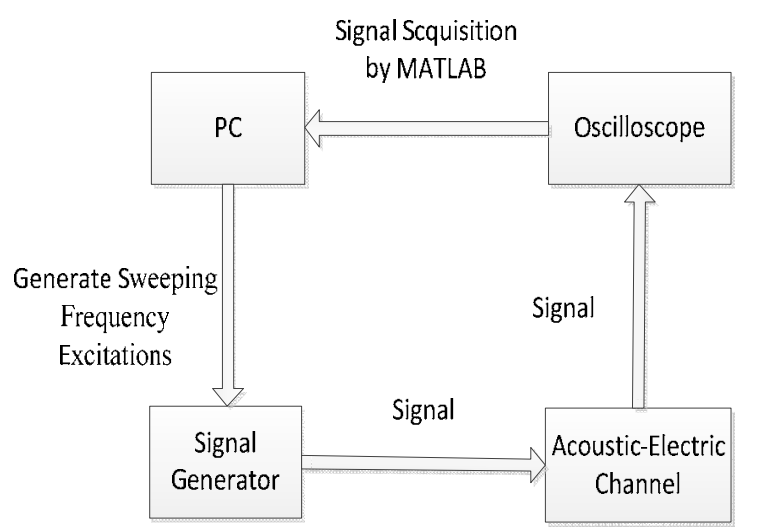

Figure 3. Operation schematic diagram of experimental process

The material properties used in the experimental are shown in Table 1.

Table 1. The material properties used in the experiment

\begin{tabular}{|c|c|}
\hline & \\
\hline Property & Value \\
\hline Density of piezoelectric $\left[\mathrm{g} / \mathrm{cm}^{3}\right]$ & 7800 \\
\hline $\begin{array}{l}\text { Area of piezoelectric } \\
\text { transducer on wall }\left[\mathrm{m}^{2}\right]\end{array}$ & 0.01 \\
\hline Wall thickness[m] & 0.022 \\
\hline $\begin{array}{l}\text { Piezoelectric transducer } \\
\text { thickness }[\mathrm{m}]\end{array}$ & 0.002 \\
\hline $\begin{array}{l}\text { Piezoelectric transducer } \\
\text { coefficient } \mathrm{e}_{33}\left[\mathrm{C} / \mathrm{m}^{2}\right]\end{array}$ & 23.2 \\
\hline
\end{tabular}

\begin{tabular}{|l|l|}
\hline $\begin{array}{l}\text { Elastic stiffness at constant } \\
\text { displacement } \mathrm{c}^{\mathrm{D}}{ }_{33}\left[\mathrm{~N} / \mathrm{m}^{2}\right]\end{array}$ & 15.87 \\
\hline Mechanical quality factor Q & 80 \\
\hline
\end{tabular}

Mason equivalent circuit model of the receiving transducer parameters and transmit transducers take the same values because they use the same type of transmit transducer.

The experimental result is shown in Fig. 4, in order to test the theory of equivalent circuit model, we compared the result of simulation and experimental, from which we can see the theoretical simulation results show good agreement with experiment results.

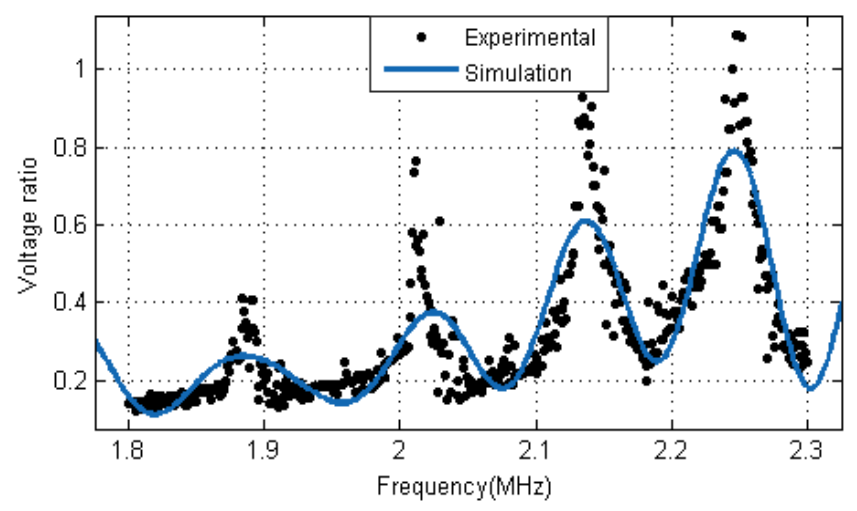

Figure 4. Simulation result compared with experimental

Measured result of signal piezoelectric transducer using a precision impedance analyzer is shown in Fig. 5, from which we can see that the minimum impedance of this kind of piezoelectric transducer is about $2.1 \mathrm{MHz}$.

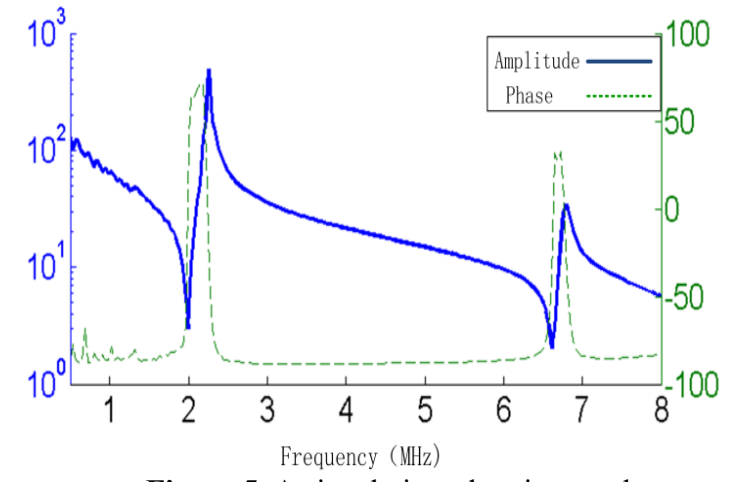

Figure 5. A signal piezoelectric transducer impedance character

Point to be noted that the physical parameters used in these models vary depending on piezoelectric transducer's statements: hanging freely in air or attached to the metal wall with epoxy or a cyanoacrylate adhesive. Piezoelectric transducer coupled with different metal wall and the result of is shown in table2.

Table 2. Results of coupled forms with different

\begin{tabular}{|l|l|l|l|}
\hline \multicolumn{4}{|c|}{ metal wall } \\
& $\begin{array}{l}\text { Intrinsic } \\
\text { frequency }\end{array}$ & $\begin{array}{c}\text { Aluminum } \\
(5 \mathrm{~mm})\end{array}$ & Steel(10mm) \\
\hline Impendence[$[\Omega]$ & 3.017 & 21.4 & 6.03 \\
\hline Frequency[MHz] & 2.101 & 2.135 & 2.138 \\
\hline
\end{tabular}


The result show effectiveness of the simulation modeling algorithm and be fit for application in the engineering. Meanwhile, wireless energy transmission using ultrasonic is feasible.

\section{Summary}

Although some important milestones were reached in the technology of wireless energy transmission using ultrasonic through metal wall with the effort of researches during recent decades, much work remains to be done in practical engineering application. The theory model must be developed further to allow for functionality capable for use in a practical design.

According to the engineering needs of wireless energy transmission through metal wall, we analyze the acoustic and electric equivalent circuit of Mason model. Based on the preliminary analysis theory of the model, we test the characteristics of through metal wall based on ultrasonic energy transmission with the experimental methods. Voltage is a measure of the energy carrier by the charge, strictly: voltage is the "energy per unit charge", thus, using voltage representing energy is reasonable.

\section{ACKNOWLEDGEMENTS}

The authors would greatly appreciate the support provided by National Nature Science Foundation of China No.51375485 and No.51305449 for this work.

\section{References}

1. Yang, Ding-Xin, et al, Through-Metal-Wall Power Delivery and Data Transmission for Enclosed Sensors: A Review. Sensors 15.12 (2015) 3158131605.

2. Lawry, Tristan J., et al, A high-performance ultrasonic system for the simultaneous transmission of data and power through solid metal barriers, Ultrasonics, Ferroelectrics, and Frequency Control, IEEE Transactions on 60.1 (2013) 194-203.

3. Wilt, K. R., Lawry, T. J., Scarton, H. A., RoaPrada, S., Saulnier, G. J., Ashdown, J. D., Das, P. K. and Pinezich, J. D. Mechanical Design Implications on Power Transfer Through Thick Metallic Barriers Using Piezoelectric Transducers, ASME 2010 International Mechanical Engineering Congress and Exposition, (2010).

4. Zhang, Q., Shi, S. and Chen, W. Research on Effective Electric-Mechanical Coupling Coefficient of Sandwich Type Piezoelectric Ultrasonic Transducer Using Bending Vibration Mode, Advances in Mechanical Engineering, 7 (1), 204370-204370, (2014).

5. Chang, Zensheu, et al, Power loss consideration in wireless piezoelectric acoustic-electric power feedthru, The 14th International Symposium on: Smart Structures and Materials \& Nondestructive Evaluation and Health Monitoring. International Society for Optics and Photonics(2007)

6. Lawry, T. J., Wilt, K. R., Roa-Prada, S., Ashdown, J. D., Saulnier, G. J., Scarton, H. A., Das, P. K. and Pinezich, J. D. Electrical optimization of power delivery through thick steel barriers using piezoelectric transducers, Proceedings of SPIE - The International Society for Optical Engineering, 7683 (6), 768314-768314-12, (2010).

7. Krimholtz, Richard, David A. Leedom, and George L. Matthaei, New equivalent circuits for elementary piezoelectric transducers, Electronics Letters 6.13 (1970): 398-399.

8. Xiao, S. and Li, Y. Modeling and High Dynamic Compensating the Rate-Dependent Hysteresis of Piezoelectric Actuators via a Novel Modified Inverse Preisach Model, IEEE Transactions on Control Systems Technology, 21 (5), 1549-1557, (2013).

9. Paradies, R. and Schläpfer, B. Finite element modeling of piezoelectric elements with complex electrode configuration, Smart Materials \& Structures, 18 (2), 1282-1294, (2009).

10. Hu, Yuantai, et al, Transmitting electric energy through a metal wall by acoustic waves using piezoelectric transducers, Ultrasonics, Ferroelectrics, and Frequency Control, IEEE Transactions on 50.7 (2003) 773-781.

11. Wilt, K. R., Scarton, H. A., Roa-Prada, S., Saulnier, G. J., Ashdown, J. D., Lawry, T. J., Das, P. K., Gavens, A. J., Wilt, K. R. and Scarton, H. A. Finite Element Modeling and Simulation of a Two-Transducer ThroughWall Ultrasonic Communication System, ASME 2009 International Mechanical Engineering Congress and Exposition, (2009). 